nephron

Practice
Nephron 2018;138:13-21

DOI: $10.1159 / 000479751$
Received: June 24, 2017

Accepted after revision: July 21, 2017 Published online: August 26, 2017

\title{
Bedside Stereomicroscopy of Fabry Kidney Biopsies: An Easily Available Method for Diagnosis and Assessment of Sphingolipid Deposits
}

\author{
Einar Svarstad ${ }^{a, d}$ Sabine Leh ${ }^{b, d}$ Rannveig Skrunes ${ }^{a, d}$ \\ Kristin Kampevold Larsen ${ }^{b}$ Øystein Eikrem ${ }^{a}$ d Camilla Tøndel ${ }^{c, d}$ \\ ${ }^{a}$ Department of Medicine, ${ }^{b}$ Department of Pathology, and ${ }^{c}$ Department of Pediatrics, Haukeland University \\ Hospital, and ${ }^{d}$ Department of Clinical Medicine, University of Bergen, Bergen, Norway
}

\section{Keywords}

Stereomicroscopy $\cdot$ Fabry disease $\cdot$ Podocyte .

Globotriaosylceramide

\begin{abstract}
Background/Aims: A previous case report found stereomicroscopic changes typical for Fabry disease in a kidney biopsy. This case series evaluates an expanded diagnostic capacity of the method. Methods: Bedside stereomicroscopy was performed in a cross-sectional prospective study of 31 consecutive enzyme-treated or treatment-naïve male ( $n=$ 14) and female Fabry disease patients. The burden of glomerular storage material was scored semiquantitatively on a visual analog scale (range 0-3) and a blinded comparison was done with a reference histologic method. Results: Significant correlations $(p<0.001)$ were found between the stereomicroscopic scoring of glomerular characteristic white storage material and the amount of podocyte globotriaosylceramide (Gb3) deposits scored by standardized light microscopy. The bedside method correctly identified the variability of podocyte $\mathrm{Gb3}$ accumulation after 10 years of identical agalsidase therapy in 2 brothers aged 24 and 27 years, and also identified tubular cell deposits. Stereomicroscopy correctly verified the absence of sphingolipid deposits in the biopsy of a female index patient with a genetic variant of unknown significance, and the diagnosis of Fabry disease was finally discarded. Conclusions: Bedside stereomicrosco-
\end{abstract}

py of kidney biopsies is an easily available, low-cost microscopy method handled by the clinician. The method carries a high diagnostic sensitivity for Fabry disease, reducing the risk of misdiagnosis in previously unknown cases. An expanded yield of the method is suggested, including the grading of the podocyte $\mathrm{Gb} 3$ burden and assessment of effectiveness of enzyme replacement therapy. We recommend the method as complementary to current standard histologic evaluation of Fabry kidney biopsies.

(c) 2017 The Author(s)

Published by S. Karger AG, Basel

\section{Introduction}

Fabry disease is a complex X-linked lysosomal storage disorder. Reduced agalsidase activity causes deposition of sphingolipids, mainly globotriaosylceramid (Gb3), in multiple renal cell types in a progressive way that eventually causes kidney failure in many patients.

Kidney pathology in Fabry disease patients has been described in detail [1]. The presence of characteristic Gb3 deposits on electron microscopy has been defined as the gold standard for diagnosis of Fabry nephropathy [2, 3]. The importance of kidney biopsies in diagnosis of Fabry disease as well as its potential in the assessment of therapeutic effect of enzyme replacement therapy has recently been highlighted $[3,4]$. Multifactorial mechanisms are involved in progressive Fabry nephropathy affecting glo-

\begin{tabular}{|c|c|c|}
\hline KARGER & $\begin{array}{l}\text { (C) } 2017 \text { The Author(s) } \\
\text { Published by S. Karger AG, Basel }\end{array}$ & $\begin{array}{l}\text { Einar Svarstad, MD, PhD } \\
\text { Department of Medicine, Haukeland University Hospital } \\
\text { PO Box } 1400\end{array}$ \\
\hline $\begin{array}{l}\text { E-Mail karger@karger.com } \\
\text { www.karger.com/nef }\end{array}$ & $\begin{array}{l}\text { This article is licensed under the Creative Commons Attribution- } \\
\text { NonCommercial-NoDerivatives } 4.0 \text { International License (CC BY- } \\
\text { NC-ND) (http://www.karger.com/Services/OpenAccessLicense). } \\
\text { Usage and distribution for commercial purposes as well as any dis- } \\
\text { tribution of modified material requires written permission. }\end{array}$ & $\begin{array}{l}\text { NO-5021 Bergen (Norway) } \\
\text { E-Mail einar.svarstad@gmail.com }\end{array}$ \\
\hline
\end{tabular}


merular, vascular and interstitial compartments, many of which are only partly understood [5]. Progressive damage of the glomerular filtration barrier plays an important role in the development of albuminuria/proteinuria and focal and segmental glomerular sclerosis in general, leading to progressive organ failure in kidney diseases, including Fabry disease $[1,2,6]$. In particular, the role of podocyte damage has been highlighted in Fabry nephropathy, and early structural and functional damage as well as podocyturia have been linked to podocyte Gb3 deposits and clinical disease in Fabry disease [7, 8]. For assessment of disease severity and renal effect of enzyme replacement therapy, histopathologic examination of glomerular, tubulointerstitial and vascular kidney biopsy compartments is pivotal. Consensus on a semiquantitative method for histologic scoring of podocyte deposits of Gb3 has been established by the International Study Group of Fabry nephropathy [9]; however, the method is currently not routinely available in all centers. Recently, an unbiased stereological quantitative method for electron microscopic assessment of podocyte Gb3-accumulation and podocyte damage has been described by Najafian et al. [7], the method is however currently used only for research purposes.

The finding of characteristic swollen glomeruli with whitish material seen during dissection-microscopic examination in a young female Fabry disease patient was first reported by Yuen et al. [10]. We have previously shown that a rapid, simple, and easily available evaluation of the kidney biopsy specimen by the nephrologist, using a bedside stereomicroscope, can diagnose glomerular deposits typical of Fabry disease in heterozygous patients [11]. The aim of the current study was to further assess the capacity of the method to score the burden of Gb3 deposits in comparison to the histologic scoring of podocyte deposits according to the International Study Group of Fabry Nephropathy [9].

\section{Material and Methods}

Bedside stereomicroscopy examination of the sharply illuminated complete biopsy cylinder was undertaken immediately after the biopsy procedure in 31 consecutive biopsies of male or female $(\mathrm{M} / \mathrm{F}=14 / 17)$ patients with either established or suspected Fabry disease. The biopsies were accrued during the period 2014-2016. The study was approved by the Regional Ethics Committee. The kidney biopsy specimen was examined after immersion in a fixative fluid ( $4 \%$ formaldehyde), and viewed against a black piece of plastic material. The illuminated biopsy cylinder floating in fixative fluid against a black background gives excellent visual contrasts, affording the clinician with a 3-dimensional impression of the diffusely distributed glomerular white deposits when subtle focusing changes are made. Digital images were taken with a Leica DFC320 camera mounted on a Leica MS5 stereomicroscope with a Leica L2 light source. The images were transferred to a computer in real-time and visualized within the Leica application suite version 4.5 software (Leica Microsystems, Wetzlar, Germany; Fig. 1). The patients were either treatment-naïve or treated by various doses of agalsidase replacement for several years. Glomerular storage material was scored semiquantitatively in the microscope (Fig. 1) on a visual-analog scale (range $0-3$ ) according to the amount and distribution of light-reflecting white deposits (sphingolipids) in the glomerulus $(0=$ absent, $1=$ minimal $[\leq 5$ white scattered spots $]$, $2=$ moderate $[6-15$ scattered spots], $3=$ severe $[>15$ spots diffusely involving the entire glomerulus]). The biopsies were scored by an experienced nephrologist, who also performed the biopsies. The transparency of the illuminated kidney tissue cylinders may show some variations, and the sphingolipid material assessed and scored by stereomicroscopy was situated in the most superficial tissue structures. If glomerular sphingolipid burden appeared to be heterogeneously distributed, the glomerulus most densely filled with sphingolipids was scored, only one score was reported for each patient. The information was stored in the patient files. The stereomicroscopy scores in this cross-sectional study were prospectively compared with subsequent scores obtained using a standardized histologic method according to the International Study Group of Fabry Nephropathy as the reference method [9]. The histologic scorings were performed by 2 experienced nephropathologists blinded to the stereomicroscopy scores. Podocyte Gb3 inclusions were scored $0-4$ in epoxy-embedded toluidine blue stained semi-thin sections, and podocyte vacuolizations were scored 0-3 in paraffin-embedded periodic acid-Schiff stained sections, accounting for all scorable glomeruli. The 2 scores were combined to a composite score 0-7 [12]. Examples of the range of glomerular stereomicroscopy scores and clinical data are given in figures from 4 patients. Statistics were performed using SPSS version 23. Glomerular stereomicroscopy scores and reference histologic glomerular scores were compared by linear regression.

\section{Results}

The stereomicroscopy set up is shown in Figure 1. The digital image of the complete biopsy specimen shows the conspicuous white-looking glomeruli typical for Fabry nephropathy. Figures $2-5$ show examples of the respective stereomicroscopy scores $0-3$ in 4 patients with different Fabry phenotypes. Patient 1, a 42-year-old female index patient with a genetic variant of unknown significance (c.490G $>$ T) previously designated as possibly damaging [13], had a diagnostic kidney biopsy as part of the clinical work-up. All glomeruli had normal reddish color and no white material was seen in any part of the biopsy specimen. A clinical stereomicroscopy score of $0 / 3$ was determined, which mirrored the subsequent histologic podocyte inclusion score of 0 (semi-thin toluidine blue section). Because of this finding, in combination with an absence of all rel- 

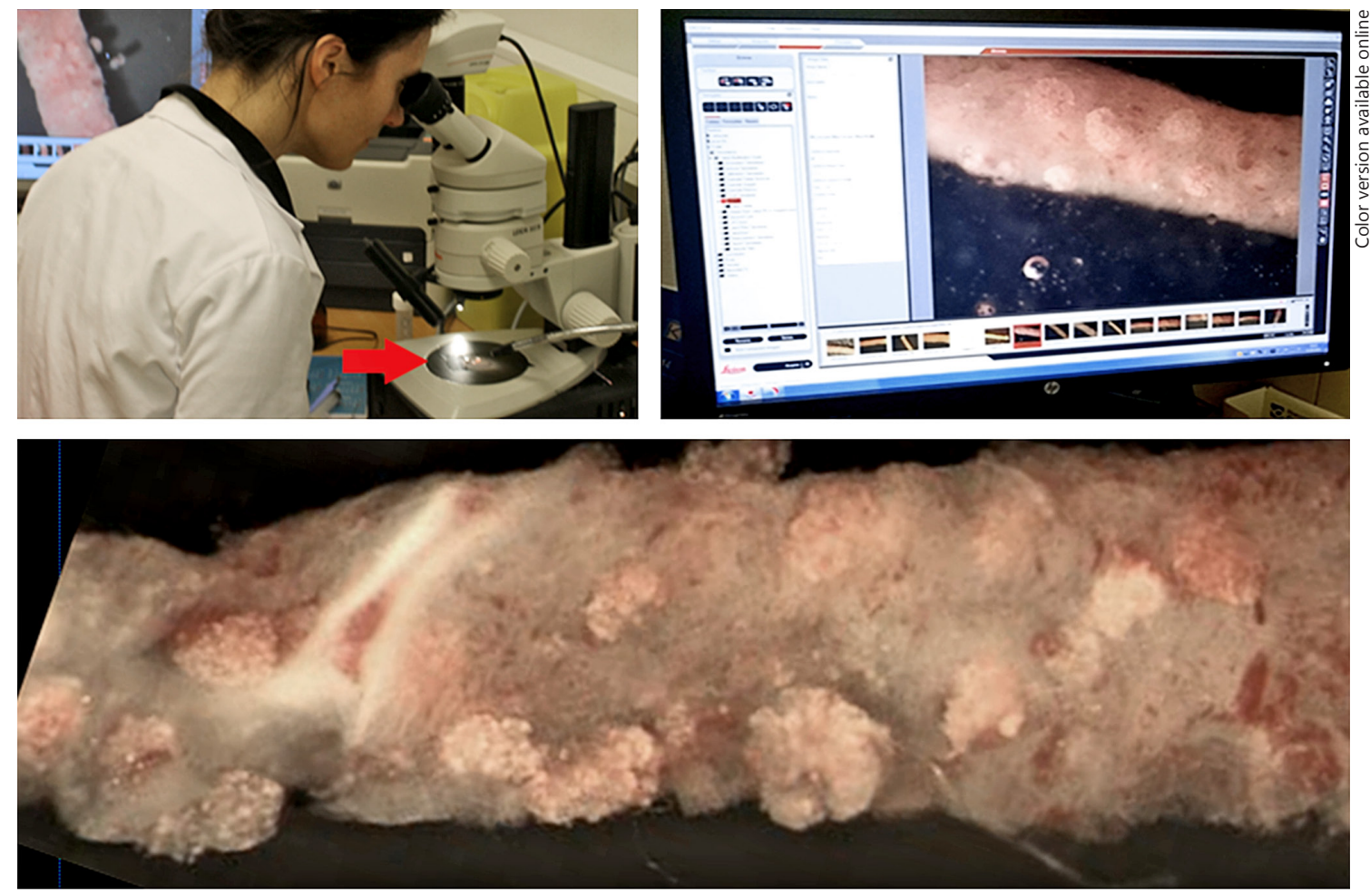

Fig. 1. The clinical bedside stereomicroscopy evaluation and scoring of glomerular deposits in the biopsy specimen (arrow) are done immediately after the biopsy procedure, and the information is stored in the patient file. A digital image is transferred to the computer (upper right panel). The lower panel shows the conspicuous "white glomeruli," which are typical findings in Fabry disease (magnification $\times 40$ ). The biopsy is from an enzyme treatment-naive classical male patient with a splice mutation (c.802-2_802$3 \mathrm{del}$ ), aged 48 years. Iohexol GFR $60 \mathrm{~mL} / \mathrm{min} / 1.73 \mathrm{~m}^{2}$, and subtle microalbuminuria. evant Fabry-related clinical findings [3], the diagnosis Fabry disease was rejected (Fig. 2). Patient 2, a 53-year-old female with a classical phenotype and disease-causing mutation $(\mathrm{c} .679 \mathrm{C}>\mathrm{T})$ had a protocol biopsy as part of routine work-up after 10 years therapy with agalsidase- $\beta$ $1.0 \mathrm{mg} / \mathrm{kg}$ every other week. Kidney function was normal with normoalbuminuria. A bedside stereomicroscopy score of $1 / 3$ was obtained. The histologic podocyte Gb3 inclusion score was 2.1/4.0 (toluidine blue semi-thin section; Fig. 3). Patients 3 and 4 were brothers aged 24 and 27 years, respectively, with classical Fabry disease (mutation 1208delAAG) having protocol biopsies as part of their general evaluation after 10 years of treatment with agalsidase- $\alpha 0.2 \mathrm{mg} / \mathrm{kg} /$ every other week. Kidney function was stable and normal with normal albumin creatinine ratio in both patients; the older brother showed slightly elevated tubular proteinuria (alpha1 microglobulin creatinine ratio $0.7 \mathrm{mg} / \mathrm{mmol}($ reference level $<0.6)$ ). The glomerular stereomicroscopy score did, however, differ in the two in spite of similar agalsidase doses (agalsidase- $\alpha$
$0.2 \mathrm{mg} / \mathrm{kg}$ every other week) and treatment time. The bedside score was $2 / 3$ in the younger brother compared to a histologic podocyte Gb3 inclusion score of 2.8/4.0 (semithin toluidine blue section; Fig. 4). The biopsy from the older brother showed uniform white glomeruli deposits corresponding to a stereomicroscopy score of $3 / 3$, which was paralleled by a histologic podocyte Gb3 inclusion score of 4.0/4.0 (semi-thin toluidine blue section; Fig. 5). In addition, the latter patient demonstrated abundant white lipid material in tubular structures (Fig. 5) not present in the biopsy from the younger brother. The discrepant findings in the siblings were also subsequently confirmed by the reference method, including the conspicuous and abundant tubular deposits seen by stereomicroscopy. Significant correlations $(p<0.001)$ were found between the clinical bedside stereomicroscopic method and the reference histologic scoring of podocyte vacuolizations (periodic acid-Schiff sections) and inclusions (toluidine blue semi-thin sections). The best correlation $\left(R^{2}=0.737\right)$ was seen using the composite score (Fig. 6). 

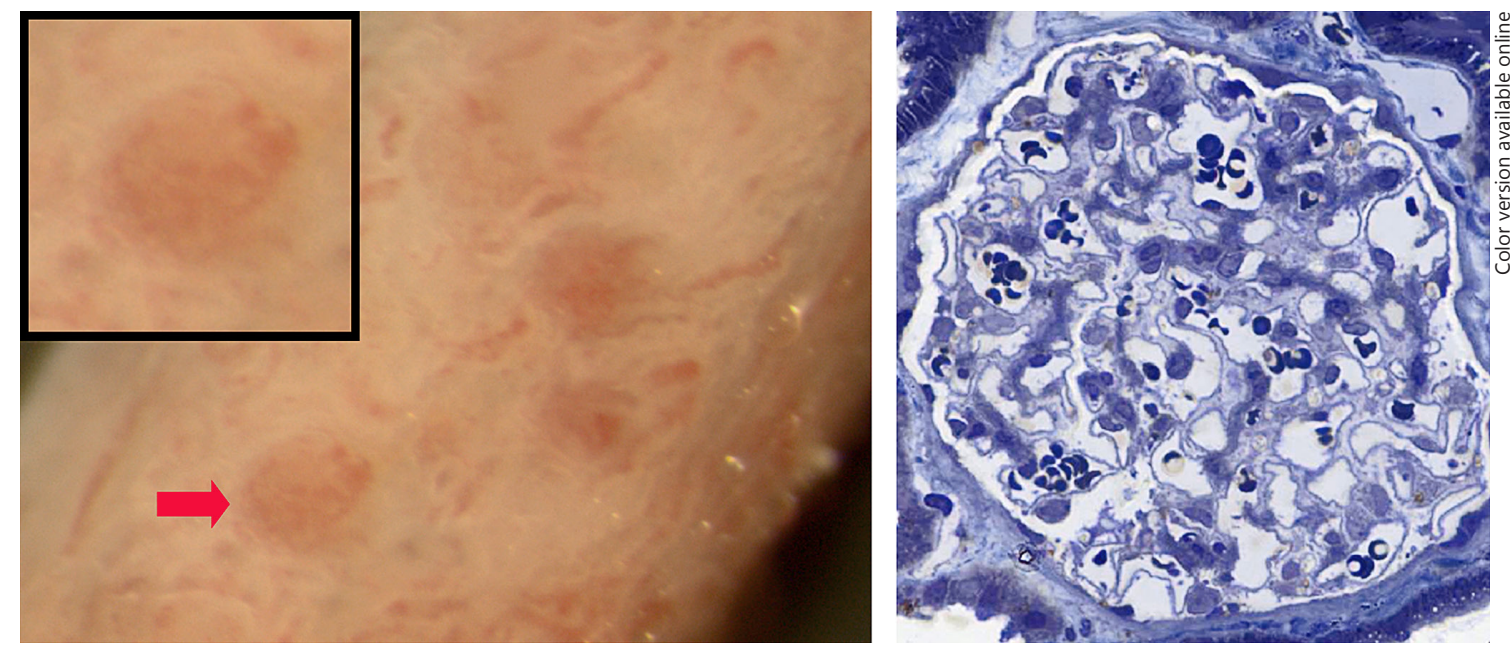

Fig. 2. Left panel shows stereomicroscopic normal red-looking glomeruli (arrow) from cortex without lipid deposits, and a stereomicroscopy deposit score $0 / 3$ (magnification $\times 40$ ). A magnifica- tion $(\times 80)$ of the glomerulus is shown in the upper square. Right panel shows a toluidine blue stained semi-thin section with no glomerular deposits (score 0/4; magnification $\times 400$ ).
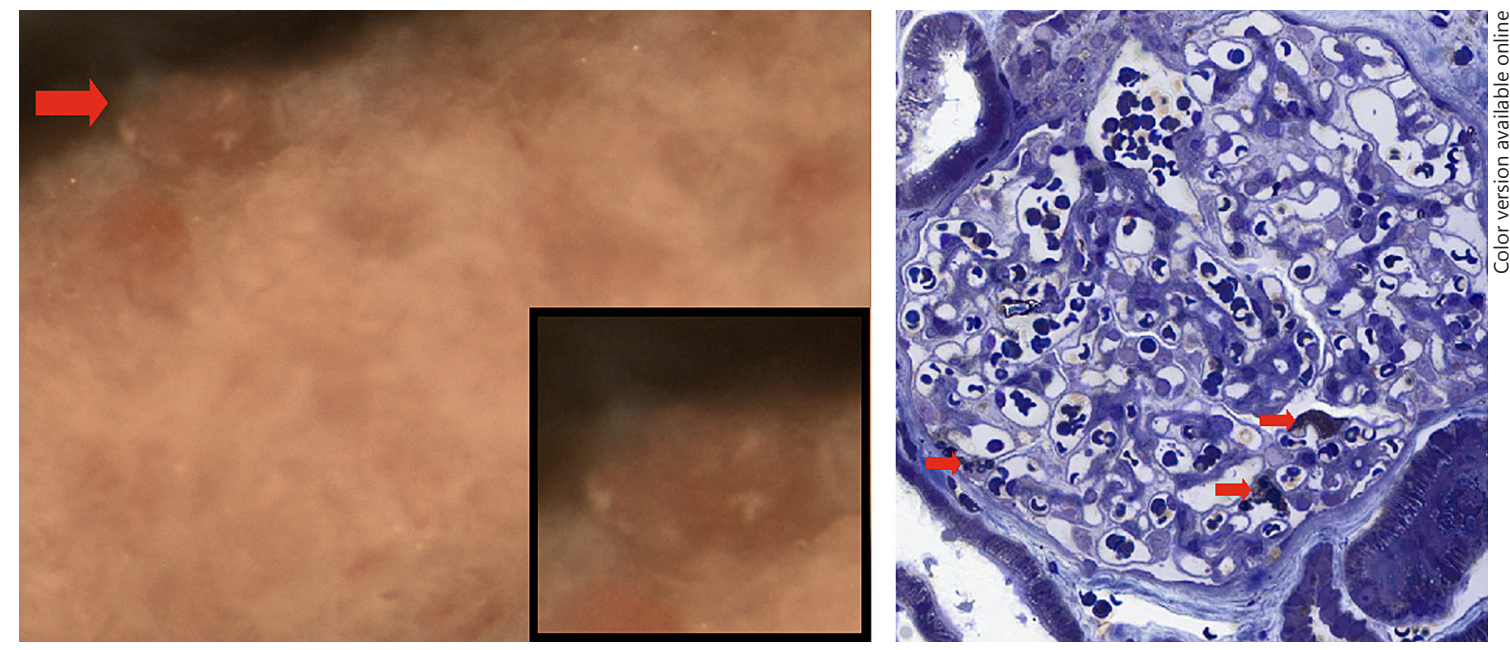

Fig. 3. Left panel shows stereomicroscopic white material scored $1 / 3$ in superficially located glomerulus (arrow; magnification $\times 40$ ), shown separately inside the square (magnification $\times 80$ ). Right panel shows Gb3 inclusions (arrows) in a toluidine blue semi-thin

\section{Discussion}

Bedside stereomicroscopy is a clinical routine method to assure an adequate number of glomeruli and secure proper handling of the biopsy core. This study is an extension of the findings of a previous report [11] and confirms that the stereomicroscopy is a sensitive and probably underused method in the diagnosis of glomerular lipid deposits in Fabry disease. Our comparative studies stained glomerulus (magnification $\times 400$ ). The mean podocyte inclusion score (mean score of inclusions in eight glomeruli) was 2.1/4.0.

have confirmed that stereomicroscopic white glomerular deposits are caused by podocyte Gb3 deposits with a high sensitivity and specificity for Fabry disease. Indeed, no false positive or false negative individuals have been found in our entire Fabry biopsy material of nearly 50 unique patients (data not shown). All biopsies from patients with Fabry disease in the present cohort show conspicuous and individually variable amounts of glomerular lipid deposits with the bedside stereomicrosco- 

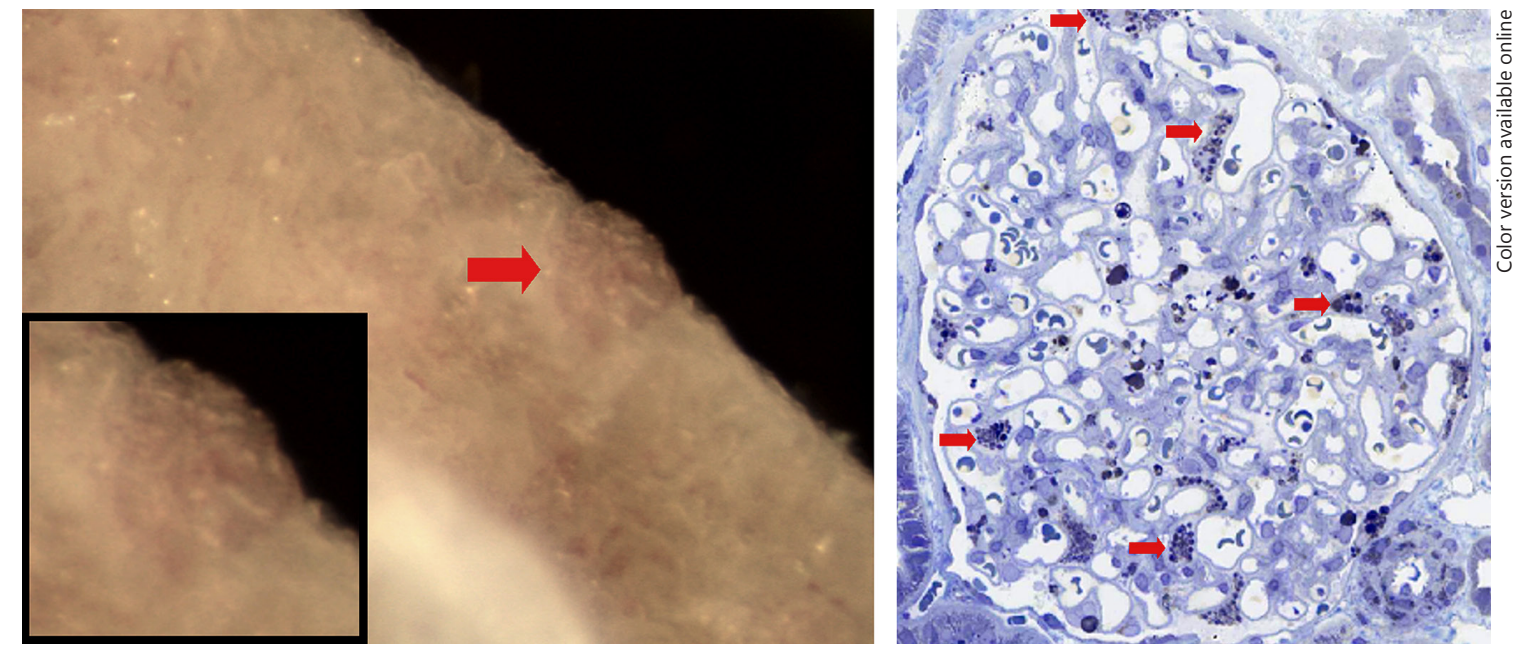

Fig. 4. Left panel shows white deposits in one superficial glomerulus (arrow) (magnification $\times 40$ ), shown separately in the lower square (magnification $\times 80$ ). The bedside stereomicroscopy score is $2 / 3$, while the mean score of Gb3 inclusions in eleven glomeruli was 2.8/4.0. Right panel shows Gb3 deposits (arrows) in toluidine blue semi-thin glomerular section of one glomerulus (magnification $\times 400)$. py, and all were subsequently confirmed by experienced nephropathologists using the reference histologic method previously described by the International Study Group of Fabry nephropathy [9]. Although the stereomicroscopy method cannot discern between the different types of glomerular cells, the overwhelming majority of the deposits have been shown to involve the podocytes. Furthermore, in patients treated with enzyme replacement therapy, the inclusions in glomerular mesangial or endothelial cells are usually cleared $[12,14]$. Consequently, for all practical purposes, the residual deposits are located in podocytes. A major novelty in the study was the capacity of the bedside stereomicroscopy method to discriminate and categorize the variability of the burden of podocyte Gb3 deposits seen in individual Fabry disease patients. Furthermore, a new observation was the finding of highly significant correlations between the stereomicroscopy scoring and the scoring of podocyte Gb3 inclusions by the reference method [9]. These observations expand the potential role of the bedside stereomicroscopy in diagnosing Fabry disease and grading the potentially reversible tissue damage in Fabry kidney biopsies.

The bedside scoring method implies obvious benefits to the clinician. First, the immediate finding of conspicuous white deposits in glomeruli, either scattered or diffusely distributed, in biopsies from patients with kidney disease of unknown etiology is likely a strong diagnostic sign of Fabry disease. The recognition of "white glomeru- lar deposits" in a particular biopsy is of great importance for an optimal result of the subsequent histopathologic examination by the nephropathologist. As Gb3 deposits are dissolved by the standard processing method for histology, where alcohol is used in several steps, such a finding may promptly lead to an adequate processing method; when the tissue samples are embedded in epoxyresin for electron microscopy the fatty deposits are conserved and Gb3 deposits are scored in toluidine blue stained semi-thin sections and electron microscopy [9]. Additional immediate reporting of the stereomicroscopic findings from the clinician to the nephropathologist may even include information about uniform (Fig. 1) or variable distribution of white material in glomeruli as well as in tubular compartments (Fig. 5). This may specifically alert the nephropathologist and increase the diagnostic yield of the histologic examination.

Second, the bedside method adds to the diagnostic armamentarium for Fabry disease that now comprises 3 different levels of tissue information. The first and immediately available level is the current stereomicroscopy method, which is a rapid, simple and low-cost procedure, and easily available to the nephrologist. The scoring uses a simple $0-3$ visual analog scale and is obtained within a few minutes. No additional technical support is needed beyond the standard stereomicroscope, equipment for digital photos and a computer for archival reasons. This is in contrast to the second-line method, which is the established light microscopy semiquantitative reference 

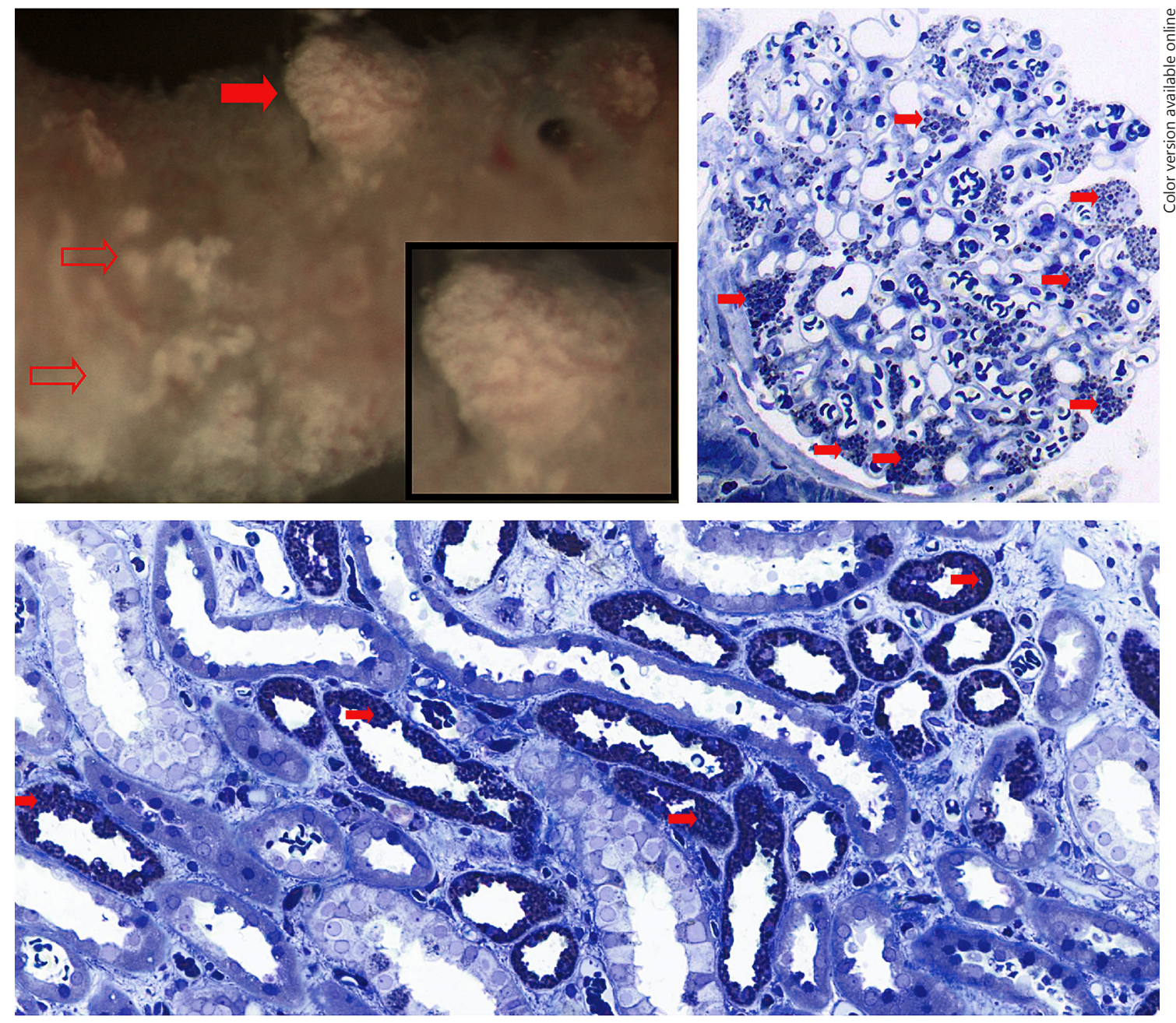

Fig. 5. Stereomicroscopy (upper left panel; magnification $\times 40$ ) shows glomerulus loaded with white material (arrow) with a full score (3/3), and also abundant white material in tubular structures (open arrows). Upper right panel (toluidine blue stained semi-thin section; magnification $\times 400$ ) shows numerous Gb3 inclusions (arrows) in a glomerulus, the mean score of inclusions in 5 scorable glomeruli was 4.0/4.0. Lower panel shows numerous Gb3 deposits in tubular cells (arrows). method [9] and the third line method is an even more detailed morphometric unbiased stereological histologic scoring method [7]. These methods are laborious, timeconsuming, expensive and highly dependent on nephropathology expertise only available in selected centers. The latter method is currently available only for research purposes. Stereomicroscopy is complementary to and should always be accompanied by the standard histology method for specific and comprehensive assessment of all kidney compartments [9], as well as electron microscopy whenever possible. An exception is kidney biopsy examinations in resource-limited countries where routine supplemental adequate histopathology methods are lacking or are performed in a delayed manner, and the bedside scor- ing system may be the only available routine diagnostic method.

Of note, case 1 in the current study (Fig. 2) showed that the absence of light reflecting white material in the glomeruli (a zero stereomicroscopy score), confirmed by a completely normal histologic examination, led to the disproving of the Fabry disease diagnosis. A recent publication has listed the current mutation (c.490G $>\mathrm{T}$ ) as potentially disease causing [13]. Our finding, therefore, clearly shows the importance of a kidney biopsy in genetic variants of uncertain pathogenicity. The current finding has important consequences for the patient, and unnecessary costly enzyme replacement therapy can be avoided. The fact that the stereomicroscopy method may give immedi- 
Fig. 6. Significant correlations $(p<0.001)$ were found between the bedside glomerular stereomicroscopy score and the reference method for histologic podocyte inclusion scores according to the International Study Group of Fabry Nephropathy [9]. The highest correlation was seen with composite score (sum of PAS and toluidine blue semi-thin sections scores).

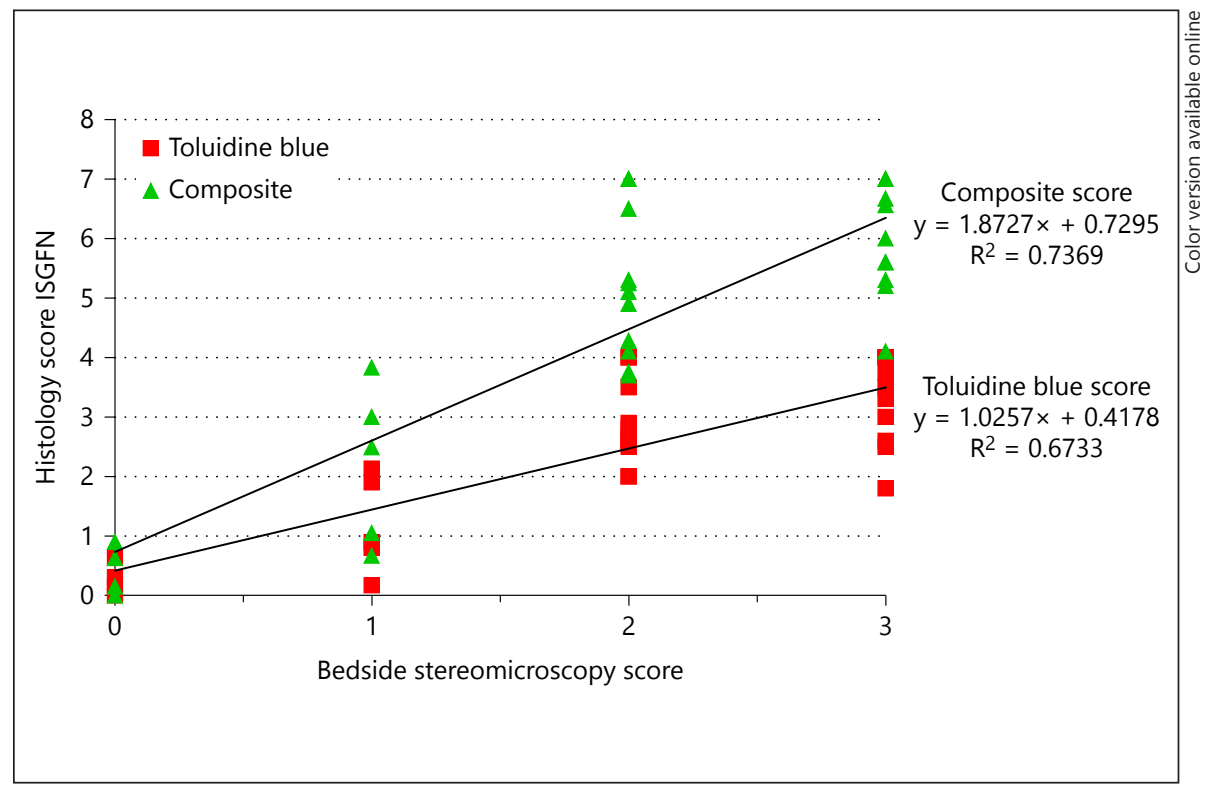

ate and adequate information helping the clinician to discern between disease-causing mutations and genetic variants of unknown significance is a new observation. After the introduction of Fabry screening programs in many countries, patients with polymorphisms and mutations with uncertain pathogenicity are more increasingly found $[13,15]$.

Cases 2-4 (Fig. 3-5) show the capacity of the stereomicroscopy scoring method to assess differences in the effectiveness of enzyme therapy, the scoring of white deposits was paralleled by similar histologic scorings of Gb3 deposits in the toluidine blue stained sections. The relevance of diagnosing the increase or decrease of podocyte inclusions has previously been shown in case reports and small series, and provides guidance for adjustments in dosing of enzyme replacement therapy $[12,16]$. Of note, complete regression of white glomerular deposits has been found in patients after long-term agalsidase treatment in parallel with near total regression of histologic scores [12].

Diagnostic biopsies should be considered before enzyme replacement therapy is decided in Fabry disease patients because some of them will show minimal or no renal $\mathrm{Gb} 3$ deposits $[3,15,17]$, indicating low risk of kidney disease. In the absence of stereomicroscopic glomerular sphingolipids, it is very unlikely that vascular or interstitial compartments are affected by Fabry-associated damage. On the other hand, non-Fabry related kidney damage causing albuminuria or reduced GFR may coexist in all Fabry patients and in genetic variants.
Co-existent disease can be diagnosed only with standard histopathologic examinations of kidney biopsies. We have previously shown that kidney biopsies performed with modern real-time ultrasound equipment are lowrisk procedures. We also strongly support that nephrologists in general advocate and strive to maintain their knowledge and hands-on experience regarding the procedure $[18,19]$. The important role of kidney biopsies in the diagnosis, classification and assessment of therapeutic efficacy in Fabry disease has recently been highlighted in several studies and case series $[3,7,12,16,20]$, as well as in a recent KDIGO conference report [4]. There is increasing evidence that accumulation of $\mathrm{Gb} 3$ in podocytes is an early marker of potential progressive Fabry nephropathy, and several studies have shown that prominent histological changes appear far earlier than clinical renal symptoms or albuminuria [21, 22]. Secondary changes in the filtration barrier may be a plausible mechanism for progressive structural and functional damage. Furthermore, the assessment of dose-dependent clearance of podocyte Gb3 inclusions in serial biopsies has been shown to contribute valuable information about treatment efficacy, as reported in the case series from our group $[12,16]$. The current report demonstrates that a simple bedside stereomicroscopic procedure handled by the clinician may add valuable diagnostic information, which ensures that the biopsy specimen is properly handled for subsequent routine histopathologic examinations, diagnostic delay is prevented, and an estimate of disease burden is provided. 


\section{Limitations}

The bedside stereomicroscopy method scores the most severely affected glomerulus, as opposed to the reference method [9], where the scoring of podocyte Gb3 is the mean value of all scorable glomeruli in the tissue specimen. Accordingly, the bedside stereomicroscopy method may have a numerically higher deposit score, especially in females where the amount of Gb3 deposits may be skewed according to random $\mathrm{X}$-inactivation. The method cannot discern between Fabry disease and other causes of Gb3 accumulation, for example, ingestion of hydrochloroquine or amiodarone. The high diagnostic sensitivity of the stereomicroscopy method only involves glomerular and tubular deposits. Supplemental standard histologic methods are necessary to characterize and assess the degree of Gb3 deposits in other renal compartments as well as non-specific interstitial and vascular lesions. The strength of the study is the consecutive inclusion of all patients with a kidney biopsy in the period, and the prospective design of the blinded comparisons between the scoring methods. Another strength of the stereomicroscopy method is the high sensitivity of the scoring of lipid material in the predilection areas (glomeruli and tubules), and the simultaneous overview of the white deposits in the entire biopsy cylinder. The 3-dimensional illuminated view of the available glomeruli, contrasting against a dark background, especially in glomeruli located near the edge of the cut cylinder, permits the as- sessment of Gb3 deposits in the whole glomerulus, as opposed to standard histologic evaluation, which usually are thin sections of parts of the glomerulus.

In conclusion, bedside stereomicroscopy of Fabry kidney biopsies is a simple, sensitive, easily available, lowcost method providing immediate diagnostic information to the clinician, and this information is of special benefit in resource-limited settings. The study suggests an expanded yield of the method including the individual assessment of Gb3 burden and responses to enzyme replacement therapy. We recommend the method as complementary to current standard histologic evaluation of Fabry kidney biopsies.

\section{Acknowledgements}

We would like to thank renal nurse Berit Sande for her excellent assistance while performing the practical kidney biopsy.

\section{Ethics Statement}

This study was approved by the Regional Ethics Committee.

\section{Disclosure Statement}

The authors have no conflicts of interest to declare.

\section{References}

1 Gubler MC, Lenoir G, Grünfeld JP, Ulmann A, Droz D, Habib R: Early renal changes in hemizygous and heterozygous patients with Fabry's disease. Kidney Int 1978;13:223-235.

2 Alroy J, Sabnis S, Kopp JB: Renal pathology in Fabry disease. J Am Soc Nephrol 2002; 13(suppl 2):S134-S138.

3 van der Tol L, Svarstad E, Ortiz A, Tøndel C, Oliveira JP, Vogt L, Waldek S, Hughes DA, Lachmann RH, Terryn W, Hollak CE, Florquin $S$, van den Bergh Weerman MA, Wanner C, West ML, Biegstraaten M, Linthorst GE: Chronic kidney disease and an uncertain diagnosis of Fabry disease: approach to a correct diagnosis. Mol Genet Metab 2015;114: 242-247.

4 Schiffmann R, Hughes DA, Linthorst GE, Ortiz A, Svarstad E, Warnock DG, West ML, Wanner C; Conference Participants: Screening, diagnosis, and management of patients with Fabry disease: conclusions from a "Kidney Disease: Improving Global Outcomes" (KDIGO) Controversies Conference. Kidney Int 2017;91:284-293.
5 Eikrem Ø, Skrunes R, Tøndel C, Leh S, Houge G, Svarstad E, Marti HP: Pathomechanisms of renal Fabry disease. Cell Tissue Res 2017;369: $53-62$.

6 Shankland SJ: The podocyte's response to injury: role in proteinuria and glomerulosclerosis. Kidney Int 2006;69:2131-2147.

7 Najafian B, Svarstad E, Bostad L, Gubler MC Tøndel C, Whitley C, Mauer M: Progressive podocyte injury and globotriaosylceramide (GL-3) accumulation in young patients with Fabry disease. Kidney Int 2011;79:663-670.

8 Fall B, Scott CR, Mauer M, Shankland S, Pippin J, Jefferson JA, Wallace E, Warnock D, Najafian B: Urinary podocyte loss is increased in patients with Fabry disease and correlates with clinical severity of Fabry nephropathy. PLoS One 2016;11:e0168346.

9 Fogo AB, Bostad L, Svarstad E, Cook WJ, Moll S, Barbey F, Geldenhuys L, West M, Ferluga D, Vujkovac B, Howie AJ, Burns A, Reeve R, Waldek S, Noël LH, Grünfeld JP, Valbuena C, Oliveira JP, Müller J, Breunig F, Zhang X, Warnock DG; all members of the
International Study Group of Fabry Nephropathy (ISGFN): Scoring system for renal pathology in Fabry disease: report of the International Study Group of Fabry Nephropathy (ISGFN). Nephrol Dial Transplant 2010; 25:2168-2177.

10 Yuen NW, Lam CW, Chow TC, Chiu MC: A characteristic dissection microscopy appearance of a renal biopsy of a Fabry heterozygote. Nephron 1997;77:354-356.

11 Svarstad E, Iversen BM, Bostad L: Bedside stereomicroscopy of renal biopsies may lead to a rapid diagnosis of Fabry's disease. Nephrol Dial Transplant 2004;19:3202-3203.

12 Tøndel C, Bostad L, Larsen KK, Hirth A, Vikse BE, Houge G, Svarstad E: Agalsidase benefits renal histology in young patients with Fabry disease. J Am Soc Nephrol 2013;24: 137-148.

13 Lukas J, Scalia S, Eichler S, Pockrandt AM, Dehn N, Cozma C, Giese AK, Rolfs A: Functional and clinical consequences of novel a-galactosidase a mutations in Fabry disease. Hum Mutat 2016;37:43-51. 
14 Thurberg BL, Rennke H, Colvin RB, Dikman S, Gordon RE, Collins AB, Desnick RJ, O'Callaghan M: Globotriaosylceramide accumulation in the Fabry kidney is cleared from multiple cell types after enzyme replacement therapy. Kidney Int 2002;62:1933-1946.

15 van der Tol L, Smid BE, Poorthuis BJ, Biegstraaten M, Deprez RH, Linthorst GE, Hollak $\mathrm{CE}$ : A systematic review on screening for Fabry disease: prevalence of individuals with genetic variants of unknown significance. J Med Genet 2014;51:1-9.

16 Skrunes R, Svarstad E, Kampevold Larsen K, Leh S, Tøndel C: Reaccumulation of globotriaosylceramide in podocytes after agalsidase dose reduction in young Fabry patients. Nephrol Dial Transplant 2017;32:807-813.
17 Houge G, Tøndel C, Kaarbøe O, Hirth A, Bostad L, Svarstad E: Fabry or not Fabry - a question of ascertainment. Eur J Hum Genet 2011; 19:1111.

18 Tøndel C, Vikse BE, Bostad L, Svarstad E: Safety and complications of percutaneous kidney biopsies in 715 children and 8573 adults in Norway 1988-2010. Clin J Am Soc Nephrol 2012;7:1591-1597.

19 Korbet SM: Nephrology and the percutaneous renal biopsy: a procedure in jeopardy of being lost along the way. Clin J Am Soc Nephrol 2012;7:1545-1547.

20 Najafian B, Tøndel C, Svarstad E, Sokolovkiy A, Smith K, Mauer M: One year of enzyme replacement therapy reduces globotriaosylceramide inclusions in podocytes in male adult patients with Fabry disease. PLoS One 2016; 11:e0152812.

21 Tøndel C, Kanai T, Larsen KK, Ito S, Politei JM, Warnock DG, Svarstad E: Foot process effacement is an early marker of nephropathy in young classic Fabry patients without albuminuria. Nephron 2015;129:16-21.

22 Wijburg FA, Bénichou B, Bichet DG, Clarke LA, Dostalova G, Fainboim A, Fellgiebel A, Forcelini C, An Haack K, Hopkin RJ, Mauer M, Najafian B, Scott CR, Shankar SP, Thurberg BL, Tøndel C, Tylki-Szymańska A, Ramaswami U: Characterization of early disease status in treatment-naive male paediatric patients with Fabry disease enrolled in a randomized clinical trial. PLoS One 2015; 10:e0124987. 